\title{
Web Omnidirectional Image Viewer for Field Event Extraction
}

\author{
Shunichi Hasegawa ${ }^{1}$, Kazuki Kobayashi ${ }^{2}$, Yasunori Saito ${ }^{2}$
}

\begin{tabular}{l}
\hline I N F O \\
Received \\
Accepted \\
Available on-line \\
Responsible Editor: \\
Keywords: \\
field event extraction, \\
omnidirectional image, field \\
monitoring system, web \\
application
\end{tabular}

\begin{abstract}
A B S T R A C T
A Web-based image viewer for observing an omnidirectional image of fields is proposed. Conventional monitoring systems are monitoring a narrow area of fields, and their image viewers are not optimized for comparing accumulated images. We implemented two functions of the viewer to compare images and conducted an experiment to evaluate their performance. The experimental results showed that Time-travel mode, which partially shows another image in a circular region on an omnidirectional image, had a high degree of usability for extracting field events from accumulated images. The proposed system can help farmers capture comprehensive, panoramic views of their farm for field management.
\end{abstract}

\section{Introduction}

Farmers constantly watch their farms and quickly respond to problems such as accidents and pathological change because high quality and consistency are required to increase the value of agricultural products. To aid farmers, some researchers have investigated methods for automatically collecting physiological and environmental data. Fukatsu (2005) and Hirafuji et al. (2005) developed a field monitoring system using sensing nodes with web server functionality. Morais et al. (2008) implemented a field data acquisition network based on a ZigBee network for precision viticulture. Sun et al. (2009) presented a field experiment of their solar-powered wireless device that includes a water content sensor, temperature sensors, an optical sensor, and a data logger. López Riquelme et al. (2009) deployed sensor nodes using a wireless sensor network to measure soil characteristics such as temperature, volumetric moisture content, and salinity, and successfully monitored a crop of ecological cabbage. Matese et al. (2009) also developed a wireless sensor network system to remotely monitor and collect micro-meteorological parameters in a vineyard. Honda et al. (2009) developed sensor data middleware as a platform to receive data from sensor networks to integrate a field monitoring system and Web GIS. Díaz et al. (2011) proposed an agricultural monitoring methodology using wireless sensor networks. Garcia-Sanchez et al. (2011) proposed an integrated wireless sensor network solution for precision agriculture to detect and identify intruders and to observe the production process. Related to these studies, several researchers are working on extracting significant information about fields such as a work log, or changes in the color or size of fruit. For example, Sugahara et al. (2008) developed a work logging system, and Kobayashi et al. (2012) developed a high-definition plant image monitoring system and Web-based image viewer to acquire plant status. Such information will be useful to identify the proper time for harvesting, especially when used in conjunction with crop color data. Motonaga et al. (2004) actually developed a digital fruit color chart using realistic fruit shapes to decide the growth stages of grapes. Using these methods to extract meaningful events from accumulated field data may lead to more efficient cultivation.

On the other hand, existing monitoring systems have several issues. First, since most of the existing field monitoring systems use web cameras or digital cameras to capture images of the field, the

\footnotetext{
${ }^{1}$ Shunichi Hasegawa

Graduate School of Science and Technology, Shinshu University

15tm522b@shinshu-u.ac.jp

${ }^{2}$ Kazuki Kobayashi, Yasunori Saito

Academic Assembly, Shinshu University

kby@shinshu-u.ac.jp, saitoh@cs.shinshu-u.ac.jp
} 
monitored areas are extremely limited. Therefore, a method for acquiring panoramic field images is required. Second, extracting meaningful events among accumulated images is both cumbersome and time-consuming because farmers must repeatedly search through and compare several images. An easier method of detecting and comparing field events is required.

In this paper, we propose a novel monitoring system that automatically collects omnidirectional images of fields, which can be viewed as a 360-degree panoramic image on a custom-designed Webbased image viewer. This enables users to observe field images showing conditions of the ground, sky, and workers' activities. The developed image viewer has a function to partially change the images to past images, which allows users to easily search and compare events through accumulated images. In this paper, we describe the architecture and ability of the developed monitoring system and discuss its functionality from the experimental results with participants.

\section{Omnidirectional Field Monitoring System}

\subsection{System Architecture}

Figure 1 shows the architecture of the omnidirectional field monitoring system, which consists of a 360-degree spherical panoramic camera (Ricoh Theta), a single board computer (Raspberry Pi) and a Web image viewer on a server computer. Theta captures a 360-degree image and automatically creates panoramic images. It is controlled by Raspberry Pi to automatically capture and transfer images to a cloud service. We use a Ruby script on Raspberry Pi and send a command to Theta to capture an image at fixed intervals.

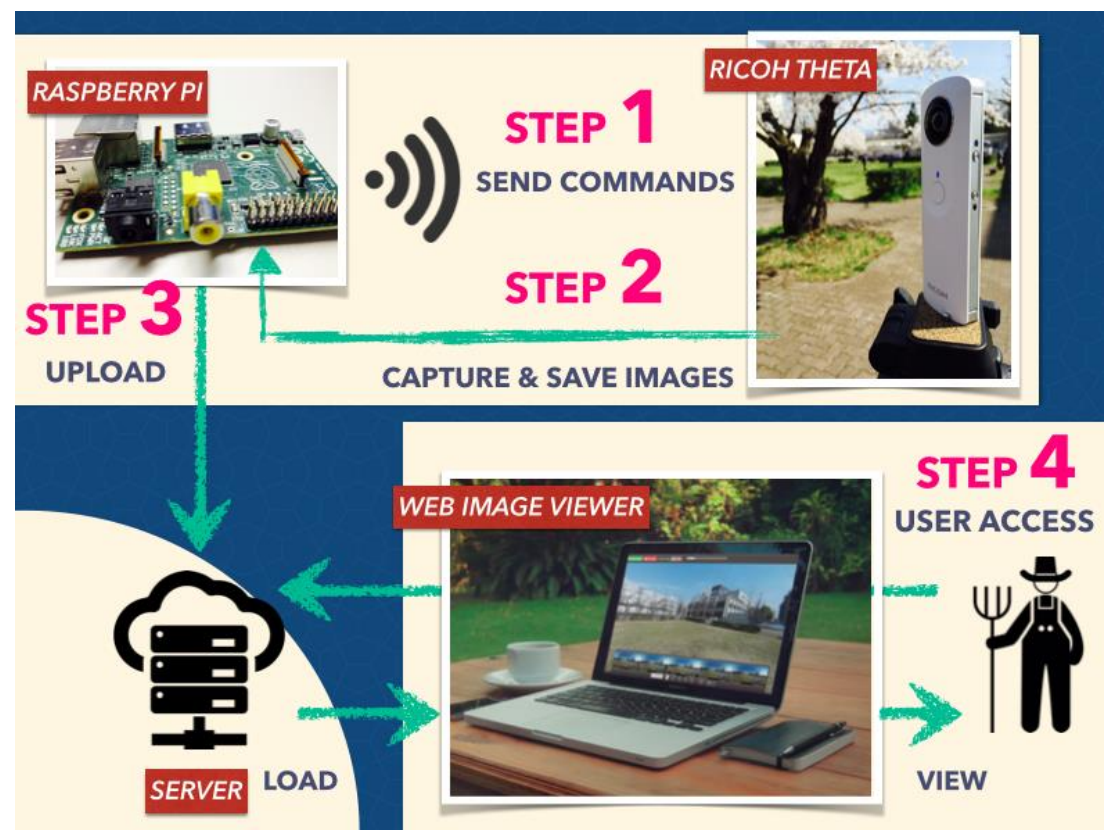

Figure 1. Architecture of omnidirectional field monitoring system

\subsection{Web Omnidirectional Image Viewer}

We developed an omnidirectional image viewer using HTML5, CSS3, JavaScript, and PHP to smoothly view a 360-degree panoramic image and extract field events on a Web browser. The image viewer was developed based on the JavaScript library, ThetaViewer, which is an open source software distributed as a jQuery plugin under MIT License. The photo taken by Theta is saved as a rectangular image (equirectangular projection), as shown in Figure 2. The viewer maps the texture on to a spherical surface using a JavaScript 3D library, Three.js. This allows the user to pan around the image with drag and drop and to zoom with the mouse wheel. 


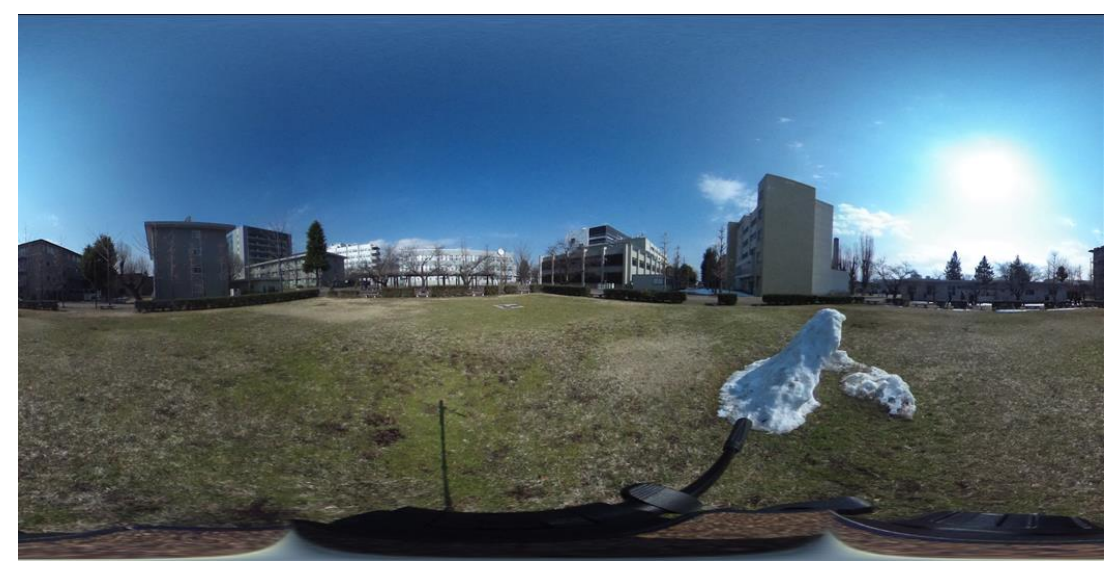

Figure 2. Original image generated by Ricoh Theta

Figure 3 shows the user interface of the developed viewer. The header of the viewer contains buttons to switch between modes that are described in a later section. The main screen shows a full panoramic image, which can be manipulated with simple mouse operations, as mentioned above. The thumbnail gallery at the bottom allows the user to select an image and change the texture to an image from a different date or time with drag and drop operation. All of the thumbnail images in the gallery are generated in the background process.

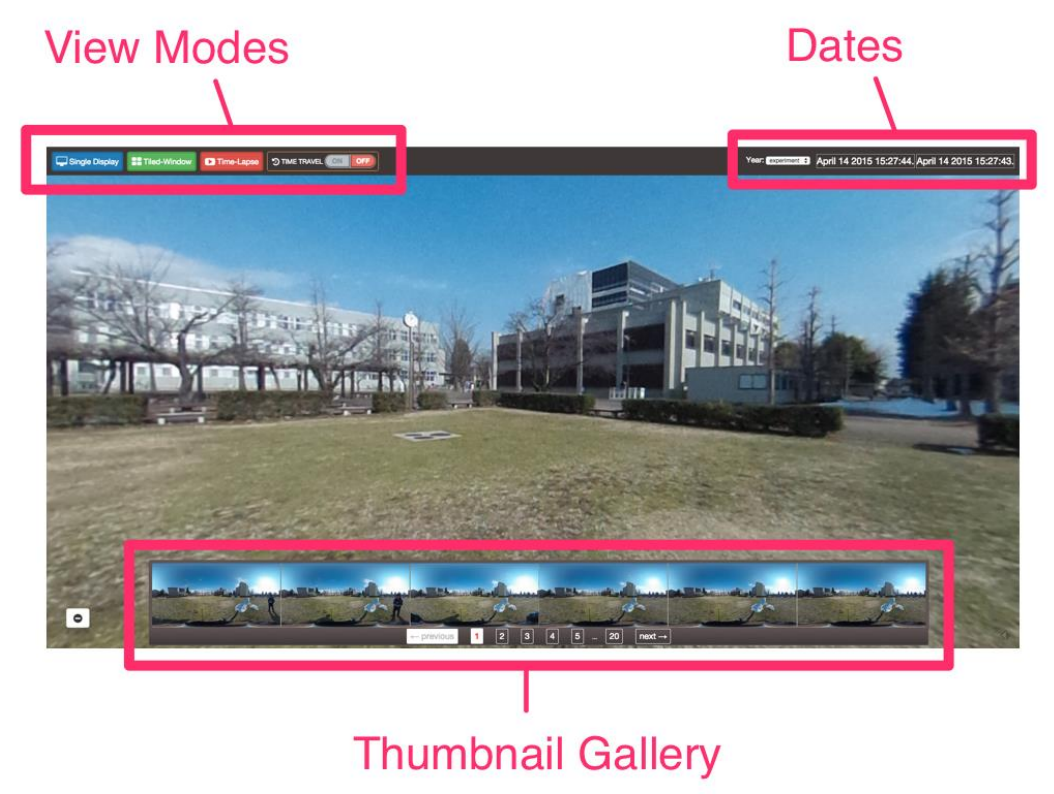

Figure 3. User interface of the Web-based omnidirectional viewer

\subsection{View Modes}

The omnidirectional image viewer has three view modes to compare field images and extract events: Tiled-window mode, Time-travel mode, and Time-lapse mode. Time-travel mode provides a novel interface to compare images. When this mode is activated, a circle appears in the middle of the screen on the base image. This circle shows a part of an image from a different date and time, and a user can freely move the circle around by dragging it. Although the screen shows one image, it actually is composed of two image layers, as indicated in Figure 4. The front layer has been clipped into a circle and an image from a different date is displayed inside. The layers share a viewpoint and zoom level so that they always synchronize while the user is manipulating the viewer. Therefore, a user can simply compare images within a single screen as they partially change the status of the environment. 
Figure 5 (left) shows the Tiled-window mode. The main screen of the Tiled-window mode is divided into four windows and arranged side by side. The user can set any image from the thumbnail gallery in the same manner as described above. This function differs from the conventional method in that it only requires single manipulation to compare images. When the viewpoint is changed by dragging and dropping, other tiled images move synchronously along with the manipulated window. It thus allows users to compare images from multiple dates with minimal effort. Figure 5 (right) shows the Time-lapse mode. To reduce the manipulation time for detecting meaningful events, we also developed another function that displays raw images taken by Theta in the modal window. It shows animation as a frameby-frame play back. In this mode, a user can grasp the entire view of the field with a glance, which helps the user specify areas to observe in detail.
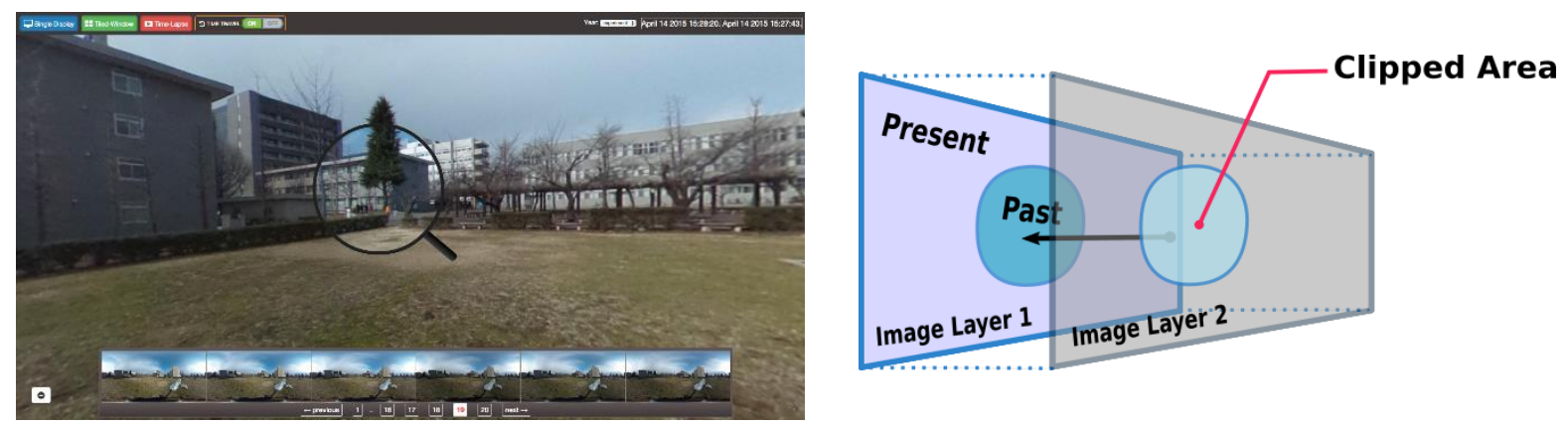

Figure 4. Time-travel mode and its layered structure
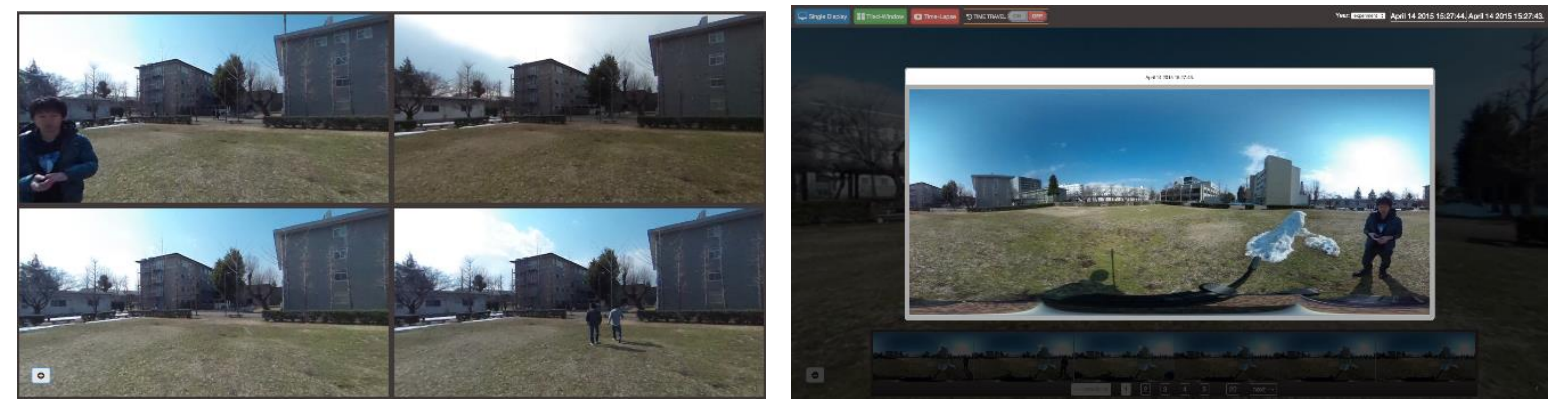

Figure 5. Tiled-window mode (left) and Time-lapse mode (right)

\section{Experiments}

\subsection{Experiment setting}

An experiment was conducted to evaluate the performance of the Time-travel and Tiled-window modes. We prepared two images for this experiment. One is a scenery image taken by Theta, and the other is a similar image with 15 subtle changes made using photo retouch software. The types of changes are classified into five categories: appearance or disappearance of an object, changes in color, changes in size, and changes in position. For example, we added copies of a window on a building, as shown in Figure 6.

We asked six participants in their early 20 s to find the differences between two images within 10 min. Half of the participants used Time-travel mode and the other half used Tiled-window mode during the experiment. In addition, participants were requested to record the areas and remaining time on a piece of paper whenever they detected a difference. 

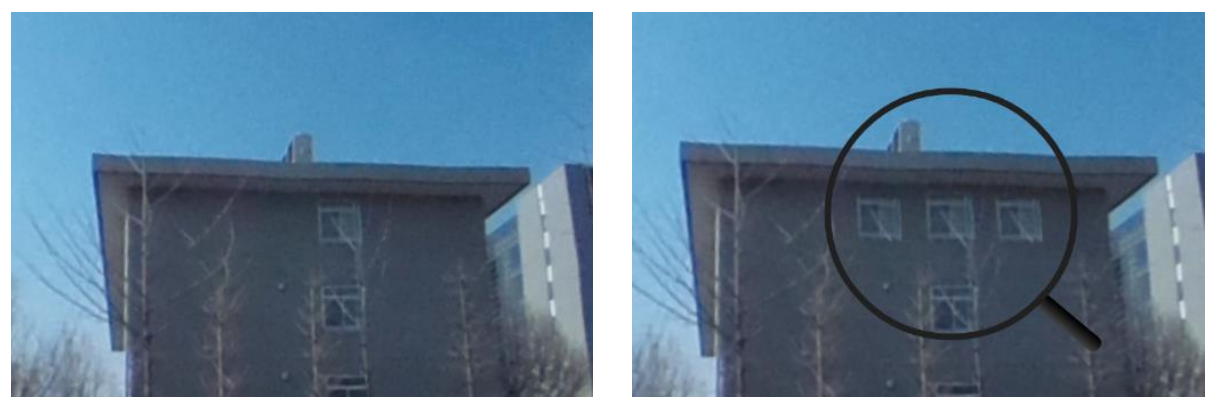

Figure 6. Example of difference between two images

\subsection{Results}

After the experiment, we calculated the average detection rate for both groups. The average detection rate for the Time-travel group was $95 \%$, which was 22 points higher than that of the Tiled-window group. Moreover, when we compared the detection rate for each item, the Tiled-window group had several items with a much lower detection rate than the Time-travel group. For instance, two items that the Tiled-window group was not able to find are highlighted in Table 1.

Table 1. Average detection rate of the differences

\begin{tabular}{|c|c|c|c|}
\hline \multirow{2}{*}{ Category } & Item & Time-Travel & Tiled-Window \\
\cline { 2 - 4 } Appearance & building & $100 \%$ & $100 \%$ \\
\cline { 2 - 4 } & window & $66 \%$ & $66 \%$ \\
\cline { 2 - 4 } & bush & $100 \%$ & $100 \%$ \\
\hline \multirow{3}{*}{ Disappearance } & snow & $100 \%$ & $66 \%$ \\
\cline { 2 - 4 } & clock & $100 \%$ & $100 \%$ \\
\cline { 2 - 4 } & manhole & $100 \%$ & $66 \%$ \\
\hline \multirow{3}{*}{ Color } & grass & $66 \%$ & $0 \%$ \\
\hline \multirow{3}{*}{ Size } & bench & $100 \%$ & $100 \%$ \\
\cline { 2 - 4 } & wall & $100 \%$ & $100 \%$ \\
\hline \multirow{3}{*}{ Position } & tree & $100 \%$ & $100 \%$ \\
\hline \multirow{2}{*}{ Total } & branch & $100 \%$ & $66 \%$ \\
\hline & emency stairs & $100 \%$ & $66 \%$ \\
\hline & garbage & $100 \%$ & $0 \%$ \\
\hline
\end{tabular}

\section{Discussion}

The experimental results showed that the Time-travel mode enabled participants to find a larger number of areas in the image that had changed. Especially, the difference in the detection rate of subtle position changes was significant. This would primarily be caused by differences in user eye movement when comparing images. In the Tiled-window mode, a user needs to go back and forth between two images to detect areas that have changed. In contrast, when a user compares images using the Timetravel mode, the user only has to move the circle over the area of interest. Therefore, the Time-travel mode not only reduces a user's workload but also helps users grasp the details of changes more precisely than the conventional method.

Additionally, the five categories that we have set up will be considered in direct relation to field events; for instance, the appearance or disappearance of an object, such as a wild animal, changes in color, such as the coloration of fruit or change of weather conditions, changes in the sizes of crops, and changes in positions, such as a farmer's activity. This information could be a great resource for making decisions on agricultural production processes. For these reasons, we believe that our developed system will help farmers extract valuable information from accumulated images and will contribute to making field management more efficient. 


\section{Conclusion}

We proposed a Web-based image viewer for viewing 360-degree panoramic images of a field. We implemented some view modes for comparison on the viewer and conducted an experiment to evaluate the performance of the modes. Time-travel mode had a high degree of usability for extracting field events from accumulated images. This suggests that the developed system can help farmers capture comprehensive, panoramic views of their farm for field management.

As a next step, we will increase the number of participants to confirm the reliability of the results. We also plan to modify the monitoring device for continuous operation in real fields because the camera of the current system cannot be charged while in capture mode and the computer system has difficulty automatically changing the modes.

\section{Acknowledgement}

This research was partially supported by the Japanese Ministry of Agriculture, Forestry and Fisheries Integration Research for Agriculture and Interdisciplinary Fields (InterACT: Interdisciplinary Agricultural Information and Communication Technology).

\section{References}

Díaz S. E., Pérez J. C., Mateos A. C., Marinescu M.-C., and Guerra B. B. 2011. A novel methodology for the monitoring of the agricultural production process based on wireless sensor networks. Computers and Electronics in Agriculture, vol.76, no.2, pp.252-265. doi: 10.1016/j.compag.2011.02.004

Fukatsu T. and Hirafuji M. 2005. Field monitoring using sensor-nodes with a web server. Journal of Robotics and Mechatronics, vol.17, no. 2, pp.164-172.

Garcia-Sanchez A.-J., Garcia-Sanchez F., and Garcia-Haro J. 2011. Wireless sensor network deployment for integrating video-surveillance and data-monitoring in precision agriculture over distributed crops. Computers and Electronics in Agriculture, vol.75, no.2, pp.288-303. doi: 10.1016/j.compag.2010.12.005

Hirafuji M., Yoichi H., Wada M., Fukatsu T., Kiura T., Shimamura H., Hu H., and Ninomiya S. 2005. Field server: multi-functional wireless sensor network node for each observation, In Proc. of the 3rd international conference on Embedded networked sensor systems (SenSys '05), page 304. doi: 10.1145/1098918.1098969

Honda K., Shrestha A., Witayangkurn A., Chinnachodteeranun R., and Shimamura H. 2009. Fieldservers and sensor service grid as real-time monitoring infrastructure for ubiquitous sensor networks. Sensors, vol.9, no.4, pp.2363-2370. doi: $\underline{10.3390 / \mathrm{s} 90402363}$

Kobayashi K., Toda S., Kobayashi F., and Saito Y. 2012. Web-based image-viewer for monitoring highdefinition agricultural images. SICE Journal of Control, Measurement, and System Integration, vol. 5, no. 1, pp.13-17.

López Riquelme J.A., Soto F., Suardíaz J., Sánchez P., Iborra A., and Vera J.A. 2009.Wireless Sensor Networks for precision horticulture in Southern Spain. Computers and Electronics in Agriculture, vol.68, no.1, pp.25-35.

Matese A., Di Gennaro S. F., Zaldei A., Genesio L., and Vaccari F.P. 2009. A wireless sensor network for precision viticulture: The nav system. Computers and Electronics in Agriculture, vol.69, no.1, pp.51-58.

Morais R., Fernandes M. A., Matos S.G., Ferreira P.J.S.G., Serôdio C., and Reis M.J.C.S. 2008. A zigbee multipowered wireless acquisition device for remote sensing applications in precision viticulture. Computers and Electronics in Agriculture, vol.62, no.2, pp.94-106.

Motonaga Y., Kondou H., Hashimoto A., and Kameoka T. 2004. A method of making digital fruit color charts for cultivation management and quality control. Journal of Food, Agriculture \& Environment, vol. 2, no.3\&4, pp. 160-166.

Sugahara K., Nanseki T., and Fukatsu T., 2008. Verification of a prototype system to recognize agricultural operations automatically based on RFID. In Proc. of Joint Conference of IAALD, AFITA and WCCA, pp. $215-$ 220.

Sun Y., Li L., Lammers P.S., Zeng Q., Lin J., and Schumann H. 2009. A solar-powered wireless cell for dynamically monitoring soil water content. Computers and Electronics in Agriculture, vol.69, no.1, pp.19-23. 